\title{
EL PLEISTOCENO INFERIOR DE LA PENÍNSULA IBÉRICA
}

\section{THE LOWER PLEISTOCENE IN THE IBERIAN PENINSULA}

\author{
por \\ EUDALD CARBONELL \\ XOSÉ PEDRO RODRÍGUEZ
}

RESUMEN Durante los últimos años se ha debatido mucho acerca de cuándo tuvo lugar el poblamiento de Europa. Los yacimientos de la Península Ibérica han aportado datos que permiten afirmar que los humanos ocuparon Europa hace más de un millón de años. En el yacimiento de Gran Dolina (Sierra de Atapuerca, Burgos) se han descubierto restos faunísticos, líticos y humanos (Homo antecessor) que datan de ca. 800,000 años. En Fuente Nueva 3 y Barranco León 5 (Granada) se han recuperado restos faunísticos y artefactos líticos que podrían tener una cronología de 1,3 millones de años. Además hay hallazgos aislados en terrazas fluviales del Noroeste, Centro y Sur de la Península que son menos concluyentes. Todos los objetos líticos recuperados pertenecen al Modo técnico 1. Por último, se discute la posibilidad de un acceso a la Península a través de Gibraltar.

ABSTRACT During the last years researches has debated about when the European settlement took place. The sites of the Iberian Peninsula have shown data that allow us to affirm that humans arrived to Europe more than one million of years ago. In the deposit of Gran Dolina (Sierra de Atapuerca, Burgos) was found a sample of faunal, lithic and human remains (Homo antecessor) dating about 800,000 years ago. In Fuente Nueva 3 and Barranco León 5 (Granada) there have recovered faunistic remains and lithic artifacts that may be able to have a chronology of 1,3 million years. In addition there are isolated findings in river terraces of the Northwest, Center and South of the Peninsula that are less conclusive. All the lithic objects recovered belong to the technical Mode 1. Finally, the possibility of an access to the Peninsula through Gibraltar is discussed.

Palabras claves Pleistoceno inferior, Gran Dolina, Fuente Nueva 3, Barranco León 5, Modo 1

Key words $\quad$ Lower Pleistocene, Gran Dolina, Fuente Nueva 3, Barranco León 5, Mode 1

ISSN: 1133-4525 ISSN-e: 2255-3924

SPAL 9 (2000): $31-47$

http://dx.doi.org/10.12795/spal.2000.i9.01 


\section{INTRODUCCIÓN}

En la década de los 1980 y principios de los 90 el paradigma dominante, controlado fundamentalmente por investigadores del centro y norte de Europa, defendía que el poblamiento humano de Europa se inició hace alrededor de 500,000 años (Gamble 1994; Roebroeks y van Kolfschoten 1994; Roebroeks et al. 1992). En aquel momento ya se conocían algunos yacimientos en Francia (Le Vallonet [Lumley et al. 1988] Soleilhac [Bonifay 1991; Bonifay et al. 1976; Bracco 1991]) e Italia (Isernia la Pineta, Monte Poggiolo) que ofrecían evidencias que podían falsear esta hipótesis. Sin embargo, algunos autores han expresado dudas acerca de estos yacimientos; se ha cuestionado la asociación entre la fauna y la industria de Le Vallonet y la cronología de Soleilhac (Jaramillo). En 1978 se descubrió el yacimiento italiano de Isernia la Pineta (Peretto 1994; 1996), en un principio adscrito al Pleistoceno inferior final, para recular luego hasta el Pleistoceno medio inicial. Poco después (en 1983) el descubrimiento de Monte Poggiolo ofrecía argumentos a quienes pensaban que los humanos vivían en Europa desde el Pleistoceno inferior final (Peretto et al. 1998). Desde los años 90 la Península Ibérica desempeña un papel fundamental en esta controversia. Mientras algunos investigadores seguían defendiendo un poblamiento iniciado en el Pleistoceno medio (Raposo y Santonja 1995), los descubrimientos de la Sierra de Atapuerca y de la Cuenca de Guadix-Baza aportaban evidencias de ocupación humana en el Pleistoceno inferior. Actualmente estos yacimientos siguen proporcionando información que nos permite afirmar, sin la menor duda, que nuestro continente ya había sido ocupado por los humanos hace más de 1 millón de años. De hecho, los descubrimientos del sur de Europa, han obligado a replantear sus teorías a quienes defendían un poblamiento reciente del continente (Dennell 1998; Dennell y Roebroeks 1996).

\section{EL PLEISTOCENO INFERIOR DE LA SIERRA DE ATAPUERCA}

En la Sierra de Atapuerca (Burgos) se han descubierto yacimientos arqueológicos y paleontológicos de cronología diversa, desde el Pleistoceno inferior hasta época histórica. Como es lógico, en este trabajo nos interesa comentar los hallazgos encuadrados en el Pleistoceno inferior. Hasta el momento hay dos yacimientos con registro arqueológico correspondiente a este periodo: Gran Dolina y Sima del Elefante. En Gran Dolina las excavaciones preliminares de los niveles 4, 5 y 6 han proporcionado registro lítico, fauna y, en el caso de TD6, fósiles humanos de más de $780 \mathrm{ka}$. Los materiales del Pleistoceno inferior de la Sima del Elefante todavía están en proceso de estudio, pero su antigüedad podría ser incluso superior a la de los de los niveles inferiores de Gran Dolina.

\section{Niveles TD4 y TD5 de Gran Dolina}

En 1990 se descubrieron en la base del yacimiento de Gran Dolina (nivel TDW4b) fauna y artefactos líticos que, según análisis paleomagnéticos posteriores, pertenecen al Pleistoceno inferior (Carbonell y Rodríguez, 1994). Los cinco artefactos hallados en TD4 fueron tallados utilizando cantos de cuarcita, obtenidos en las inmediaciones de la propia Sierra. Entre estos objetos destaca una Base Negativa de $1^{\mathrm{a}}$ Generación de Explotación (BN1GE) unifacial con escasas extracciones. Esta matriz fue utilizada como núcleo para extraer Bases Positivas (BP). El método de talla consistió en golpear repetidamente uno de los planos naturales de un espeso canto de cuarcita (talla longitudinal ortogonal). Cerca de este objeto apareció otra BN1G unifacial, probablemente también de explotación, que únicamente muestra 3 extracciones. También contamos con un canto de cuarcita (Base natural) con dos fracturas (probablemente ocurridas al intentar explotarlo), y con dos Bases Positivas: un resto de talla de reducidas dimensiones $(14 \times 11 \times 3,5 \mathrm{~mm})$ y una BP con talón cortical (Carbonell y 
Rodríguez 1994; Rodríguez Álvarez 1997). Junto a esta industria lítica (recuperada en una superficie de 7m²) apareció una rica fauna (tabla 1) (Made 1998; Rodríguez 1997; Rosell 1998; Rosell et al. 1998).

Según Rosell et al. (1998) la existencia de grandes fisuras en el techo de la cueva representaría una trampa natural para los herbívoros: los animales caerían en el interior y morirían. El descubrimiento de carcasas y huesos enteros ( $10 \%$ de los restos) así como de algunas conexiones y semiconexiones anatómicas apoyarían esta hipótesis. Los carnívoros, sobre todo los cánidos, accederían a la cueva por conductos secundarios, para nutrirse de cadáveres de herbívoros. Los humanos también habrían accedido a la cueva y habrían intervenido sobre las carcasas de los animales caídos. Así lo atestiguan las piezas de industria y la presencia de algunos huesos con fracturas antrópicas. Los restos habrían sido luego transportados por una corriente hídrica de escasa fuerza, que no sería lo suficientemente importante como para producir una desarticulación de los restos en conexión anatómica o para separar las epífisis de las diáfisis durante el transporte (Rosell et al. 1998).

También se conoce la existencia de industria lítica en el nivel TD5. De este nivel proviene una BN1GE de cuarcita, recogida del corte estratigráfico para evitar su caída. Esta Base fue objeto de una explotación unifacial sistemática, que afectó a casi todo su perímetro. También en este caso se aprovechó la morfología de la Base, particularmente el espesor, para explotarla mediante extracciones de ángulo semiabrupto.

\begin{tabular}{|c|c|c|c|}
\hline TAXONOMÍA & TD4 & TD5 & TD6 \\
\hline Stephanorhinus etruscus & & & \\
\hline Bison sp. & & & \\
\hline Bovini cf. Bison boigtstedtensis & & & \\
\hline Equus sp. (de tipo stenoniense) & & & \\
\hline Equus cf. altidens & & & \\
\hline Eucladoceros giulii & & & \\
\hline Cervus elaphus aff. acoronatus & & & \\
\hline Cervidae indet. & & & \\
\hline Dama nestii? vallonetensis & & & \\
\hline Mammuthus sp. & & & \\
\hline Sus scrofa & & & \\
\hline Mustela palerminea & & & \\
\hline Ursus praearctos & & & \\
\hline Ursus sp. & & & \\
\hline Panthera gombaszoegensis & & & \\
\hline Felidae indet. & & & \\
\hline Crocuta crocuta & & & \\
\hline Canidae indet. & & & \\
\hline Canis mosbachensis & & & \\
\hline Vulpes praeglacialis & & & \\
\hline Lynx sp. & & & \\
\hline Homotherium latidens & & & \\
\hline Homo antecessor & & & \\
\hline
\end{tabular}

Tabla 1: Macrofauna de los niveles inferiores de Gran Dolina (García y Arsuaga 1999; Made 1999; Rosell et al. 1998) 


\section{Nivel TD6 de Gran Dolina}

En julio de 1994 comenzaron a aparecer en el estrato "Aurora" del nivel TD6 restos humanos, fauna e industria lítica (Carbonell et al. 1995; Carbonell et al. 1999c). Los análisis paleomagnéticos (Parés y PérezGonzález 1995; 1999) y las dataciones por Uranio y ESR (Falguères et al. 1999) permiten afirmar que la antigüedad de estos materiales se remonta al final del Pleistoceno inferior, probablemente alrededor de los $800 \mathrm{ka}$.

En el estrato "Aurora" se han recuperado, en apenas $7 \mathrm{~m}^{2}$ de excavación y en $25 \mathrm{~cm}$ de espesor, más de 1000 restos óseos de macrofauna (tabla 1), 92 fósiles humanos y 268 artefactos líticos (Carbonell et al. 1998). TD6 ha proporcionado también un rico conjunto de micromamíferos, que ha permitido identificar 26 especies. Los arvicólidos presentes son Mimomys savini, Microtus seseae, Stenocranius gregaloides, Terricola arvalidens, Iberomys huescarensis, Allophaiomys chalinei y Pliomys episcopalis. Según Cuenca et al. (1999) el conjunto de microfauna es típico del final del Pleistoceno inferior y el inicio del Pleistoceno medio. Al inventario de la fauna hay que sumar cerca de 100 restos de avifauna, con 26 taxones identificados. La avifauna indica la existencia de un lago o una corriente poco rápida de agua, en un hábitat abierto y con unas condiciones climáticas que podrían ser similares a las de los inviernos actuales (Sánchez-Marco 1999). La información derivada de los estudios polínicos señala que el nivel TD6 presenta un porcentaje moderado de polen de robles y de encinas/quejigos, más bajo en la parte inferior del nivel. También tienen importancia las cupresáceas (sabinas, enebros, cipreses), típicas de ambientes áridos y fríos, pero su abundancia disminuye de la parte inferior a la superior de TD6. Precisamente en el estrato Aurora (situado en la parte superior del nivel TD6), las cupresáceas han disminuido bastante respecto a la parte baja de TD6, y aparecen especies de clima mediterráneo como el olivo silvestre o el lentisco. Por tanto, en TD6 se aprecia el paso desde unas condiciones frías y secas a otras más cálidas y húmedas (García Antón 1998).

En la fauna de TD6 hay variedad de especies, edades y tamaños, aunque con predominio de los individuos juveniles. El número de carnívoros, tanto en términos de número de restos como de individuos, es muy pequeño (García y Arsuaga 1999). El estudio tafonómico indica que los restos no fueron transportados por agentes naturales desde otro lugar. No hay ningún rastro de orientación preferencial de los restos ni ningún sesgo en la muestra que permita hablar de algún tipo de selección por procesos geológicos. Tampoco se observa ninguna preservación diferencial debida a causas físico-químicas. En conclusión, hay una considerable homogeneidad en el conjunto fósil, con mínimas alteraciones postdeposicionales, a excepción de fracturas diagenéticas. La representación esquelética sugiere que los animales de pequeño tamaño, incluido Homo, fueron transportados completos a la cueva, indicando un acceso primario a las carcasas. En general todos los restos (incluidos los humanos) presentan un elevado número de marcas de corte ("cut marks") y fracturas de origen antrópico, lo que indica una intensa acción sobre las carcasas que se puede relacionar con una intensa explotación de los nutrientes. Desde el punto de vista zooarqueológico, se ha interpretado este estrato como un lugar de consumo (Díez et al. 1999).

Los 92 fósiles humanos identificados en TD6 corresponden como mínimo a 6 individuos. Las edades de muerte oscilan entre los 3-4 años del individuo 6 y los poco menos de 20 años de los individuos 4 y 5. Estos fósiles presentan una combinación de caracteres que, según Bermúdez de Castro et al. (Bermúdez de Castro et al. 1997) permiten reconocer una nueva especie del género Homo. En este sentido, la morfología de los dientes es muy primitiva pero la de la cara es similar a la de las poblaciones humanas modernas. Esta nueva especie ha sido denominada Homo antecessor y representaría el antecesor común de Homo neanderthalensis y de las poblaciones modernas (Homo sapiens) (Bermúdez de Castro et al. 1997). Entre los restos destaca un neurocráneo, que ha permitido estimar una capacidad craneal de algo más de $1000 \mathrm{~cm}^{3}$.

La constatación de la existencia de canibalismo ha convertido a TD6 en un depósito excepcional. De los 92 restos humanos recuperados, casi la mitad presentan cortes producidos por útiles líticos. Estas estrías se encuentran en la mayor parte del esqueleto. También se han identificado numerosas fracturas antrópicas. 
Se da la circunstancia de que los rasgos de aprovechamiento de fauna y humanos son muy similares, por ello se ha hablado de un canibalismo "gastronómico" o alimenticio (Fernández-Jalvo et al. 1996; 1999).

\begin{tabular}{|l|rr|r|r|r|r|r|r|r|r|r|r|r|r|r|r|r|r|r|}
\hline & \multicolumn{2}{|c|}{ BN } & \multicolumn{2}{|c|}{ BN1GC } & \multicolumn{2}{|c|}{ BN1GE } & \multicolumn{2}{|c|}{ BP } & \multicolumn{2}{|c|}{ BN2GC } & \multicolumn{2}{c|}{ BN2GE } & \multicolumn{2}{|c|}{ FRAG. } & \multicolumn{2}{|c|}{ INDET } & TOTAL \\
\hline Sílex neógeno & 0 & $0,0 \%$ & 0 & $0,0 \%$ & 8 & $6,5 \%$ & 64 & $52,0 \%$ & 1 & $0,8 \%$ & 3 & $2,4 \%$ & 4 & $3,3 \%$ & 43 & $35,0 \%$ & 123 & $47,9 \%$ \\
\hline Sílex cretácico & 0 & $0,0 \%$ & 0 & $0,0 \%$ & 3 & $9,4 \%$ & 17 & $53,1 \%$ & 12 & $37,5 \%$ & 0 & $0,0 \%$ & 0 & $0,0 \%$ & 0 & $0,0 \%$ & 32 & $11,9 \%$ \\
\hline Cuarcita & 7 & $16,7 \%$ & 0 & $0,0 \%$ & 3 & $7,1 \%$ & 27 & $64,3 \%$ & 5 & $11,9 \%$ & 0 & $0,0 \%$ & 0 & $0,0 \%$ & 0 & $0,0 \%$ & 42 & $15,7 \%$ \\
\hline Arenisca & 7 & $30,4 \%$ & 0 & $0,0 \%$ & 1 & $4,3 \%$ & 12 & $52,2 \%$ & 0 & $0,0 \%$ & 0 & $0,0 \%$ & 2 & $8,7 \%$ & 1 & $4,3 \%$ & 23 & $8,6 \%$ \\
\hline Caliza & 5 & $17,9 \%$ & 1 & $3,6 \%$ & 2 & $7,1 \%$ & 16 & $57,1 \%$ & 4 & $14,3 \%$ & 0 & $0,0 \%$ & 0 & $0,0 \%$ & 0 & $0,0 \%$ & 28 & $10,4 \%$ \\
\hline Cuarzo & 0 & $0,0 \%$ & 0 & $0,0 \%$ & 1 & $5,0 \%$ & 9 & $45,0 \%$ & 2 & $10,0 \%$ & 0 & $0,0 \%$ & 8 & $40,0 \%$ & 0 & $0,0 \%$ & 20 & $7,5 \%$ \\
\hline TOTAL & 19 & $7,1 \%$ & 1 & $0,4 \%$ & 18 & $6,7 \%$ & 145 & $54,1 \%$ & 24 & $9,0 \%$ & 3 & $1,1 \%$ & 14 & $5,2 \%$ & 44 & $16,4 \%$ & 268 \\
\hline
\end{tabular}

Tabla 2 : Materias primas y categorías estructurales de la industria lítica recuperada en el estrato "Aurora” del nivel TD6 de Gran Dolina. BN= Bases naturales (cantos sin tallar, utilizados como percutores); BN1GC= Bases Negativas de $1^{\text {a }}$ Generación de Configuración (instrumentos configurados sobre canto); BN1GE = Bases Negativas de $1^{\text {a }}$ Generación de Explotación (núcleos sobre canto o bloque); $\mathrm{BP}=$ Bases Positivas (lascas); $\mathrm{BN} 2 \mathrm{GC}=$ Bases Negativas de $2^{\mathrm{a}}$ Generación de Configuración (instrumentos configurados sobre lasca); BN2GE= Bases Negativas de $2^{\text {a }}$ Generación de Explotación (lascas utilizadas como núcleo); Frag.= Fragmentos; Indet.= Indeterminables (objetos no determinables debido a su mal estado de conservación) (Carbonell et al. 1999b).

En el estrato Aurora se han recuperado 268 artefactos, tallados utilizando sílex, cuarcita, arenisca, cuarzo y caliza (tabla 2). El sílex se ha dividido en dos grupos petrológicos, en función del origen de su formación: cretácico y neógeno. Ambos pueden localizarse en un radio no superior a $5 \mathrm{~km}$ desde Gran Dolina. El sílex arqueológico aparece con frecuencia deteriorado (especialmente el de origen neógeno), con su estructura interna no cohesionada. La fuente primordial de captación de cuarcita, arenisca y cuarzo fue el río Arlanzón. La caliza se obtuvo en el propio karst, aunque también hubo una mínima selección de caliza en forma de cantos rodados, paralela a la selección de cuarcitas y areniscas. Estas materias primas pudieron obtenerse en una área que apenas dista 1 o $2 \mathrm{~km}$ del yacimiento (Carbonell et al. 1999b). Existe una clara preferencia en la utilización del sílex, sobre todo neógeno, seguido a bastante distancia por la cuarcita.

La aplicación del análisis de categorías estructurales del Sistema Lógico Analítico (Carbonell et al. 1992; Rodríguez Álvarez 1997) ha permitido constatar la preponderancia de las Bases Positivas (lascas), con más de la mitad de los efectivos (casi el 65\% si no tenemos en cuenta las piezas indeterminables). Cabe destacar la presencia de 21 núcleos (Bases Negativas de Explotación), de los cuales 18 son sobre canto o bloque (BN1GE) y 3 sobre lasca de gran formato (BN2GE). Ello supone un 7,8\% del total, que si eliminamos del cómputo global los objetos indeterminables alcanza un $9,4 \%$. Un porcentaje similar al de instrumentos sobre lasca (BN2GC), con 24 efectivos. Es interesante señalar el destacado número de cantos sin tallar (Bn), muy probablemente utilizados como percutores, tanto para tallar como para fracturar huesos. Sin embargo, solamente hemos localizado un instrumento configurado sobre canto rodado (BN1GC) (Carbonell et al. 1998).

En cuanto a las estrategias para la producción de artefactos, domina la talla ortogonal, con dos métodos: el multifacial, básicamente aplicado al sílex, y el método longitudinal, aplicado casi exclusivamente a la cuarcita (Carbonell et al. 2000; Carbonell et al. 1999b; Rodríguez Álvarez 1997). En la explotación ortogonal, el ángulo entre el plano sobre el que se percute y el plano de lascado se acerca a los 90 grados. Este tipo de gestión de la materia prima es utilizado sistemáticamente con el sílex neógeno; la matriz obtenida suele tener una morfología paralelepípeda, subcúbica o cúbica. La explotación a través del método ortogonal multifacial (creando múltiples caras en la matriz) (Figura 1) permite la obtención de productos de talla con una amplia variabilidad, tanto morfológica como de tamaño. En el caso de la cuarcita, el método ortogonal es diferente, ya que se usa la plataforma natural de los cantos como plataforma de percusión, desde la cual 
se realizan los levantamientos. Éstos normalmente atraviesan todo el espesor del canto, y se localizan en series de dos o tres extracciones. De ahí que hablemos del método ortogonal longitudinal.
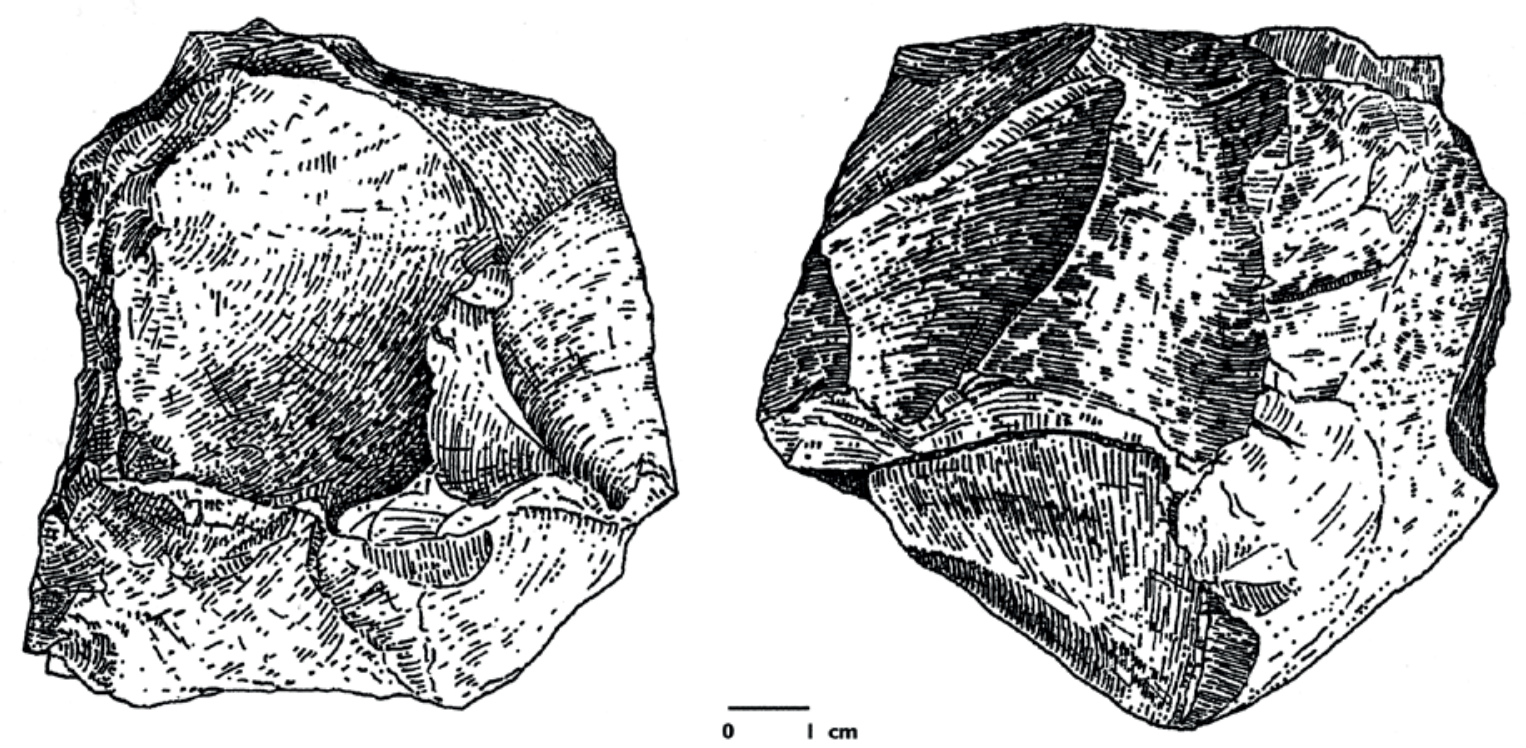

Figura 1: Base Negativa de $1^{a}$ Generación de Explotación de sílex neógeno, procedente del estrato "Aurora" del nivel TD6 de Gran Dolina. El método de explotación aplicado es multifacial ortogonal.

Los productos característicos de la explotación son las Bases Positivas con talones no facetados (que conservan el córtex original de la roca) y caras ventrales rectas - propias de la ortogonalidad de la percusión-, con porcentajes de córtex altos para materiales de origen fluvial y escaso número de nervaduras en la cara dorsal. También se ha identificado el método de explotación centrípeto, aunque su utilización es escasa.

Al menos una parte de la industria fue tallada en el interior de la cueva, ya que hemos recuperado objetos pertenecientes a todas las fases implicadas en las secuencias de talla: desde núcleos hasta restos de talla. Además hemos identificado remontajes: dos BP de sílex neógeno remontan con una BN2GE. Se da la circunstancia de que una de las BP apareció fracturada en dos piezas (halladas en el mismo cuadro). En primer lugar se remontaron estas dos piezas y luego se pudo comprobar que la BP resultante remontaba a su vez con un núcleo sobre lasca de gran formato (Rodríguez Álvarez 1997).

La representación de BN2GC (instrumentos sobre lasca) es significativa (10\%), debido primordialmente a la gran proporción de esta categoría estructural en sílex cretácico (Figura 2). Ello es reflejo de un uso diferencial de algunos materiales, las BP de sílex cretácico son especialmente seleccionadas para retocarlas y convertirlas en BN2GC (el 37,5\% de los objetos de esta variedad de sílex son lascas retocadas). Las series de retoques para la configuración del instrumental son cortas, realizadas con escaso número de levantamientos con los que se configuran fundamentalmente denticulados y, en menor medida, retoques continuos (sobre todo raederas) (Figura 2).

Es importante destacar la ausencia de grandes lascas para configurar instrumentos de gran formato tales como bifaces, hendedores, picos, etc. característicos del Modo 2. Por otra parte, en este estrato solamente hemos localizado un instrumento sobre canto (BN1GC), tallado sobre caliza. 


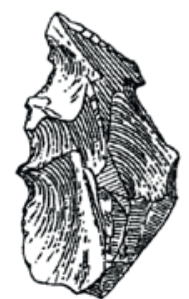

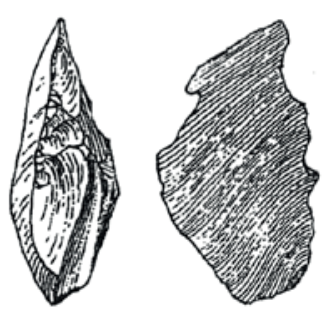

$\mathbf{a}$

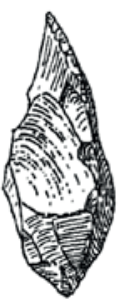

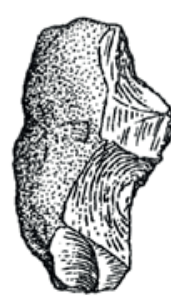
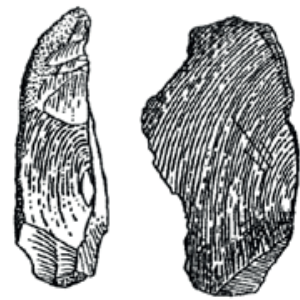

b

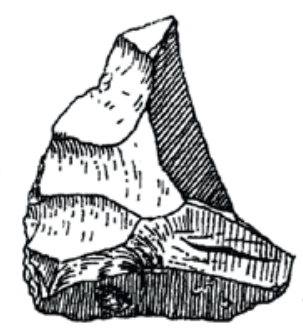

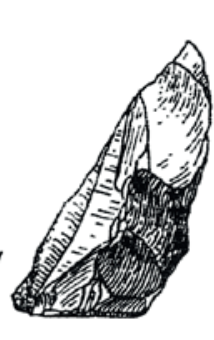

c
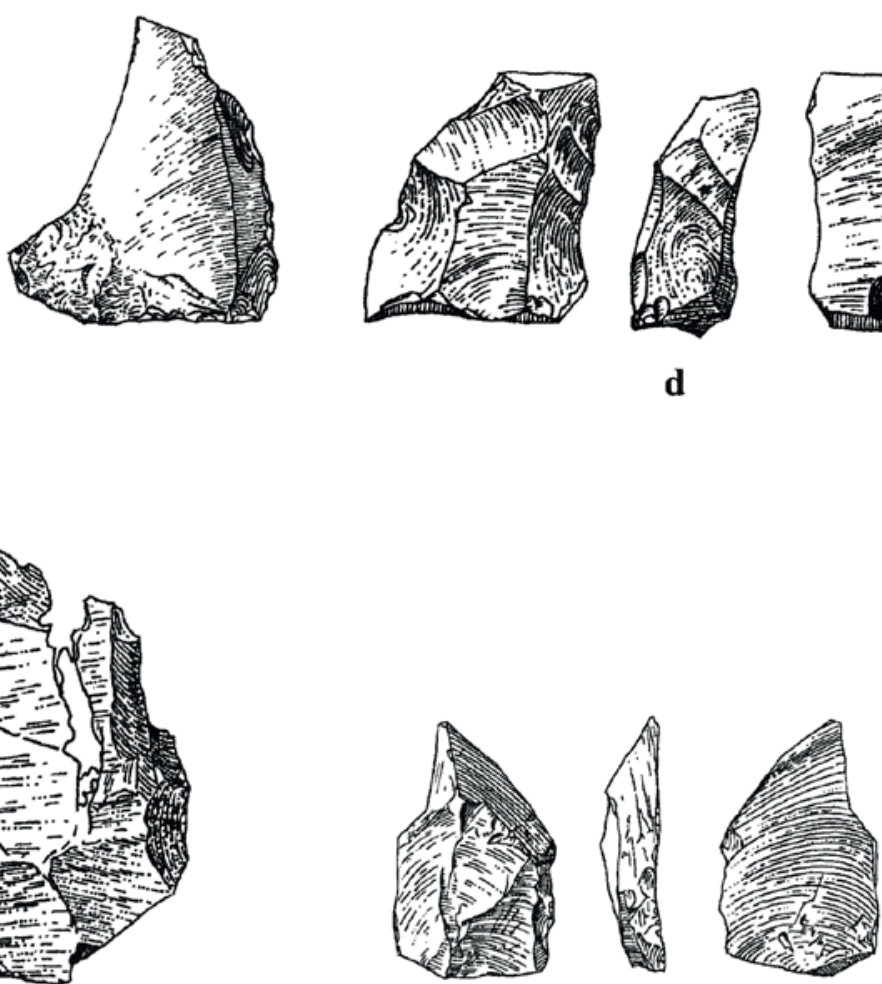

f
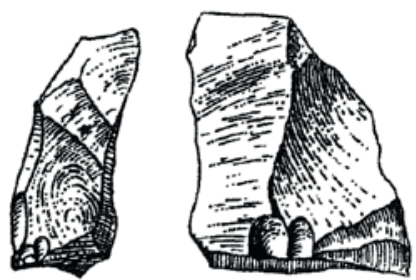

d
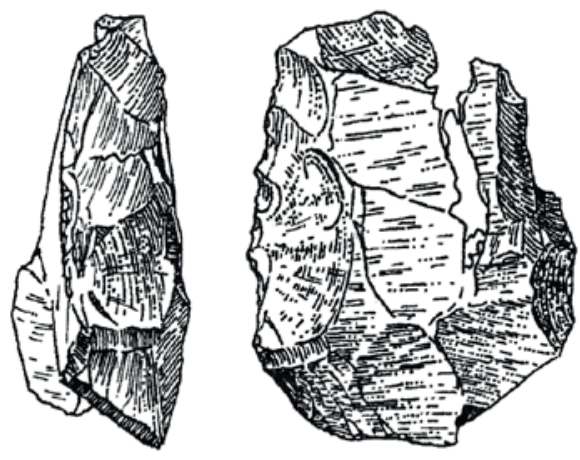

e

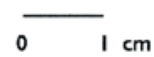

Figura 2: Bases Negativas de 2a Generación de Configuración (BN2GC), de TD6 (estrato "Aurora"); a, b, c y d = denticulados de sílex cretácico; e = retoque continuo lateral, sobre sílex neógeno deteriorado; $\mathrm{f}=$ retoque continuo lateral, sobre sílex cretácico. 
El análisis mediante Microscopio Electrónico de Barrido ha permitido identificar la función de algunos instrumentos. Se ha podido inferir que algunos instrumentos fueron utilizados en el procesamiento de carne. Un segundo grupo de actividades no tienen como objetivo el consumo de alimentos sino que se relacionan con el trabajo sobre materia dura vegetal: se trata de acciones de rascado y raspado que sólo se pueden vincular a la producción de nuevos instrumentos (Sala 1997; 1998).

La total ausencia de grandes instrumentos bien configurados e intensamente elaborados, unido al tipo de retoque aplicado y a los métodos de talla predominantemente ortogonales, apuntan a una tecnología poco compleja, de características similares al Modo 1 (Carbonell et al. 1997; 1998; 1999b; 2000).

Cuando fue ocupada por los homínidos, la cueva de Gran Dolina debió ser una de las bocas de karst más importantes de la Sierra de Atapuerca. La cueva se abría hacia el oeste, resguardada de los vientos de norte y su ubicación en una pequeña hondonada, debió inducir a Homo antecessor a escogerla como lugar en donde realizar diversas actividades. En el momento de la ocupación la bóveda de la cueva se hallaba a más de 10 metros de altura, constituyendo un espacio amplio, con luz y buenas condiciones de habitabilidad. Desconocemos por ahora su profundidad, pero sí sabemos que la zona ocupada podía tener unos 15 metros de anchura (Carbonell et al. 1998).

\section{Los yacimientos de la Cuenca de Guadix-Baza}

La Cuenca de Guadix-Baza (Granada) es una zona muy rica en yacimientos paleontológicos, con una secuencia que abarca desde el Mioceno hasta el Pleistoceno superior. La mayoría de los yacimientos se sitúan en la Formación de Baza, constituida sobre todo por sedimentos lacustres.

La existencia de yacimientos paleolíticos se conoce desde los años 60 y sobre todo 70, cuando se excavaron los yacimientos mesopleistocenos de Solana del Zamborino (Botella et al. 1975) y Cúllar Baza I (Ruiz Bustos 1984). En 1983 se descubrieron los primeros artefactos líticos en el yacimiento de Barraco León 5 y a principios de los años 90 aparecieron artefactos en Fuente Nueva 3 (Gibert et al. 1998a). A mediados de los años 90 se realizaron intervenciones en estos dos yacimientos, pero hasta 1999 no se han podido reemprender de nuevo las excavaciones (Toro 1999).

Aunque los trabajos arqueológicos en esta zona se han visto injustamente lastrados por la polémica acerca del supuesto carácter antrópico de determinados fósiles hallados en el yacimiento paleontológico de Venta Micena (Gibert et al. 1998b; Moyà-Solà y Köhler 1997; Palmqvist 1997), siempre ha habido coincidencia en la gran antigüedad de los artefactos líticos procedentes de Barranco León 5 y Fuente Nueva 3 (Gibert et al. 1998a; Martínez Navarro et al. 1997; Oms et al. 2000b; Turq et al. 1996).

La presencia de magnetización inversa a lo largo de las secciones estratigráficas de estos dos yacimientos, combinada con el conjunto faunístico, indica inicialmente una edad Matuyama para los dos sitios arqueológicos, esto es, entre 0,78 y 1,77 millones de años. La presencia de Allophaiomys bourgondiae en Fuente Nueva 3, una especie más antigua que el Microtus nivaloides de Le Vallonet (adscrito al subcron Jaramillo, entre 0,99 y 1,07 m.a.), indicaría que FN3 tiene una datación de más de 1,07 m.a. En cuanto a Barranco León 5, su situación estratigráfica podría estar ligeramente por debajo que la de Fuente Nueva 3 (Oms et al. 2000a; 2000b).

Por tanto, en función de la macro y microfauna y de los análisis palemagnéticos se estima una antigüedad de entre 1,3 y 1,1 millones de años para estos dos yacimientos (Toro 1999). 


\begin{tabular}{|l|rr|rr|rr|rr|r|}
\hline & \multicolumn{2}{|c|}{ BNE } & \multicolumn{2}{c|}{ BP } & \multicolumn{2}{c|}{ BN2GC } & \multicolumn{2}{c|}{ FRAGS } & TOTAL \\
\hline FN3 superficie & 3 & $5,6 \%$ & 33 & $61,1 \%$ & 15 & $27,8 \%$ & 3 & $5,6 \%$ & 54 \\
\hline FN3, nivel 1 & & & 2 & $100,0 \%$ & & $0,0 \%$ & & $0,0 \%$ & 2 \\
\hline FN3, nivel 2 & 1 & $2,0 \%$ & 39 & $79,6 \%$ & 5 & $10,2 \%$ & 4 & $8,2 \%$ & 49 \\
\hline FN3, nivel 3 & 1 & $33,3 \%$ & 2 & $66,7 \%$ & & $0,0 \%$ & $0,0 \%$ & 3 \\
\hline BL5 & 4 & $3,7 \%$ & 71 & $66,4 \%$ & 13 & $12,1 \%$ & 19 & $17,8 \%$ & 107 \\
\hline
\end{tabular}

Tabla 3: Industria lítica de los yacimientos de Fuente Nueva 3 (FN3) y Barranco León 5 (BL5), según Oms et al. (2000b). BNE= Bases Negativas de Explotación (núcleos); BP= Bases Positivas (lascas y restos de talla); BN2GC= Bases Negativas de $2^{\text {a }}$ Generación de configuración (lascas retocadas); Frags= Fragmentos.

\section{Barranco León 5}

Las evidencias arqueológicas y paleontológicas aparecen en un nivel de arenas finas de aproximadamente $35 \mathrm{~cm}$ de espesor, que correspondería a la parte distal de un pequeño sistema aluvial (Arribas y Palmqvist 1999b). Oms et al. (2000b) mencionan el hallazgo (hasta 1999) de 107 objetos, la mayoría tallados con sílex (sólo se menciona un objeto de cuarcita y otro de dolomía). El sílex se pudo obtener en un área próxima al yacimiento.

La mayoría del material lítico se compone de BP (más de la mitad de las cuales son de pequeño formato, restos de talla), aunque es significativo el 12\% de BN2GC (lascas retocadas) (tabla 3). El método de explotación aplicado a los 4 núcleos (BNE) es centrípeto, sin preparación de las matrices. El formato de los núcleos es pequeño (en torno a $43 \mathrm{~mm}$ de longitud media [Gibert et al. 1998a]). Los productos resultantes son BP con talones fundamentalmente corticales, lisos y en pocas ocasiones facetados. En el mismo nivel apareció una rica fauna que indica claramente una cronología de Pleistoceno inferior (Oms et al. 2000b).

\section{Fuente Nueva 3}

La sección de Fuente Nueva 3 se compone primordialmente de una sucesión de calizas, limos blancos calcáreos y lutitas. Los artefactos líticos se hallaron en superficie y en estratigrafía, sobre todo en el nivel 2 . Según Oms et al. (2000b) la sedimentación indica un entorno de baja energía. La fauna es numerosa y claramente del Pleistoceno inferior. La ausencia de marcas de dientes en la fauna y la casi ausencia de carnívoros excluiría una actividad de predadores en su acumulación. Entre los 54 artefactos encontrados en superficie, al menos 45 tienen concreción de color rojizo, similar a la que aparece en los artefactos hallados en el nivel 2, lo que podría indicar que estos artefactos también pertenecían a este nivel (Oms et al. 2000b). En cualquier caso, se cita la existencia de 49 objetos en el nivel 2, de los que casi el $80 \%$ son Bases Positivas (más de la mitad, 29, de pequeño formato)(tabla 3). Cabe destacar un 10\% de BN2GC según los datos proporcionados por Oms et al. (2000b), aunque Martínez-Navarro et al. (Martínez Navarro et al. 1997) señalan que son muy escasas las lascas retocadas. Entre las BN2GC se han reconocido raederas, denticulados y muescas. La materia prima utilizada son pequeños cantos de sílex de entre 10 y $15 \mathrm{~cm}$ de longitud. Oms et al. (2000b) piensan que puede estar representada toda la cadena operativa, aunque sólo se ha identificado un núcleo (BNE). Si tenemos en cuenta todo el material, podemos deducir que se utilizaron diversos métodos de talla (Martínez Navarro et al. 1997; Turq et al. 1996): el más frecuente consiste en series cortas de extracciones con cambios relativamente frecuentes de plataforma de percusión; también aparece la talla 
centrípeta; y por último un método de explotación ortogonal unipolar unilineal, que da lugar a lascas alargadas, de tendencia laminar. Este tipo de explotación se realizaría utilizando la técnica bipolar, que produce la aparición de BP con dos bulbos y frecuentes fracturas longitudinales ("accidentes de Siret"). Generalmente los talones son corticales, planos y raramente facetados, aunque se menciona la existencia de algunos talones convexos multifacetados.

\section{OTRAS EVIDENCIAS}

Aparte de los yacimientos de Atapuerca y Guadix-Baza hay otras localizaciones que podrían aportar datos sobre el poblamiento inicial de la Península Ibérica. En Cueva Victoria (Murcia) se descubrió una falange que podría ser humana, aunque no se puede descartar completamente la posibilidad de que pertenezca a Theropithecus oswaldi, del que se ha hallado en este yacimiento un molar. La citada falange podría provenir de una brecha datada en más de 1 millón de años (Palmqvist et al. 1996), de ser así, nos encontraríamos ante el fósil humano más antiguo de la Península.

Por otro lado, hay algunos hallazgos de artefactos líticos en terrazas fluviales que podrían remontarse al final del Pleistoceno inferior o al inicio del Pleistoceno medio. Las adscripciones cronológicas de estos yacimientos se realizan en función de la posición y las características morfoestratigráficas de las terrazas. Desgraciadamente, la imposibilidad de conseguir dataciones absolutas, la carencia de restos faunísticos y la escasez de los restos líticos limita mucho la información.

Las investigaciones realizadas desde los años 90 en el sur de Galicia han permitido reconstruir el proceso de sedimentación del tramo final del río Miño, de manera que se han reconocido 8 niveles de terrazas. En algunas de las terrazas altas se ha localizado industria lítica que, según Giles et al. (2000), podría remontarse al límite entre el Pleistoceno inferior y el medio. La industria localizada en la terraza más alta (T8, +80 metros) en Peteira (Tuy) es muy escasa y procede del pie del corte estratigráfico. También se menciona el hallazgo de una BN1GC en conexión estratigráfica en la T7 (+65/72 m) en Tomiño-Campo de Fútbol (Pontevedra). En Chan de Vide (Setado, Pontevedra), se ha localizado industria en conexión estratigráfica en las terrazas T7 y T5 (+42/55 m), en los yacimientos de Minas del Condado 5 (T7) y Minas del Condado 3 (T5). También hay industria en la T6, pero no en estratigrafía, sino al pie del corte. La industria de Minas del Condado 5, que podría pertenecer al final del Pleistoceno inferior, consta de BN1GE, BN1GC (unifaciales y bifaciales) y BP de cuarcita. En Minas del Condado 3 aparecieron también BN1GE, BN1GC y BP en cuarcita muy alterada. También hay hallazgos en superficie en Montes de Oleiros (municipios de Salvaterra do Miño y de As Neves), recogidos al pie del corte estratigráfico de la T5, con BN1GE, BN1GC y BP de cuarcita. En general estas industrias constan de instrumentos configurados sobre cantos de gran tamaño (BN1GC). En Minas do Condado también se han identificado Bases negativas para la producción de BP de gran formato. Los investigadores que han descubierto estas industrias, las adscriben al Modo 1 (Giles et al. 2000: 111).

En la Meseta se han citado hallazgos en terrazas fluviales que podrían pertenecer al Pleistoceno inferior final y al Pleistoceno medio inicial. En la Meseta sur los artefactos más antiguos, escasos y pendientes de confirmación según Santonja y Pérez-González (Santonja y Pérez-González 1997), proceden del nivel de +105 metros del Tajo, en Pueblanueva. Aquí se localizaron 2 objetos: una BN1GC, una BN1GE agotada y un fragmento proximal de BP. Los materiales recogidos en la terraza de $+60 / 65 \mathrm{~m}$ del río Sangrera, en San Bartolomé de las Abiertas, podrían pertenecer a comienzos del Pleistoceno medio. En este lugar se documentan 18 objetos: 5 BN1GE (dos de las cuales son discoides), 8 BP y 5 BN2GC (Santonja y PérezGonzález 1997). Los materiales de El Espinar (en la terraza de +75/80 m del Tajo) son, según Santonja y Pérez-González, más dudosos. 
En la Submeseta norte se han recogido artefactos en las terrazas del Duero, en los niveles de +80 metros (en Toro) y +74 (en la confluencia de Valderaduey). También se han documentado hallazgos aislados en las terrazas de +60 y +80 del Pisuerga, en el entorno de Valladolid (Rojo y Moreno 1979). En la terraza de 70 metros del Esla, en Perilla del Castro y Bretocino, se descubrieron BN1GE y fragmentos de BP. También pueden ser muy antiguas las piezas encontradas en la terraza de $+50 / 54$ metros del Tormes, en la zona de La Maya III. Todos estos hallazgos corresponderían a momentos que van desde el final del Pleistoceno inferior a comienzos del Pleistoceno medio. Dada la escasez de hallazgos se hace difícil la adscripción de esta industria a un Sistema Técnico específico, pero podrían pertenecer perfectamente al Modo 1.

En el sur de la Península, el estudio detallado de las terrazas fluviales del Bajo Guadalquivir ha permitido establecer una secuencia que comienza con los complejos de terrazas muy altas (T1 a T9, que abarcarían el Pleistoceno inferior y parte del Pleistoceno medio), terrazas medias (T10 a T12, resto del Pleistoceno medio) y terrazas bajas y muy bajas (T13 y T14, del Pleistoceno medio final y superior al Holoceno) (Vallespí Pérez 1994). La obtención de dataciones ha permitido enmarcar cronológicamente esta secuencia de terrazas. Entre T3 y T4 se ha determinado el evento Jaramillo (entre 0,99 y 1,07 millones de años), en T10 se ha identificado el evento Biwa $(280 \mathrm{ka}$ ) y por último hay una datación de $80 \mathrm{ka}$ (Uranio/Torio) para la T12. Las industrias más antiguas proceden de la T6, mientras que en las localizaciones de la T8 aparecen los primeros bifaces. Por tanto, es probable que los artefactos más antiguos se sitúen en el Pleistoceno medio inicial.

En la parte Oeste de la Península se registró desde los años 40 la presencia de industrias de cantos tallados muy antiguas, localizadas en la Estremadura portuguesa (Breuil y Zbyszewski 1942-1945). Casi siempre se trataba de hallazgos de superficie, recogidos en lo alto de antiguas playas. Algunos de estos hallazgos se adscribieron al Pleistoceno inferior. Sin embargo, una revisión crítica de estos materiales, utilizando criterios geológicos, geomorfológicos y tipológicos, hace recaer serias dudas sobre las antiguas adscripciones (Raposo y Carreira 1986).

\section{EL PRIMER POBLAMIENTO DE LA PENÍNSULA IBÉRICA}

Los datos obtenidos de los yacimientos de la Cuenca de Guadix-Baza y de la Sierra de Atapuerca, permiten afirmar que la Península Ibérica fue ocupada por humanos (probablemente Homo antecessor) hace algo más de un millón de años. Por otro lado, los hallazgos dispersos de artefactos en las terrazas de algunos ríos del centro de la Península y del Noroeste, podrían complementar los hallazgos de Atapuerca y GuadixBaza. Por consiguiente, se observa un poblamiento significativo durante el Pleistoceno inferior final, desde luego más significativo que durante el Pleistoceno medio inicial, ya que hasta hace alrededor de 500-400 ka. no se aprecia una intensificación de la ocupación.

Aunque no es el tema central de este artículo, es necesario hacer referencia al origen de los grupos humanos que colonizaron la Península durante el Pleistoceno inferior. Tal y como señalamos al principio, la cuestión del primer poblamiento de Europa es objeto en los últimos años de numerosos trabajos que abordan la cuestión desde diversos puntos de vista: cuándo se produjo, por qué se produjo y cómo (o, más exactamente, por dónde) se produjo (Aguirre y Carbonell 2001; Arribas y Palmqvist 1999a; Bar-Yosef y Belfer-Cohen 2001; Carbonell 1999; Carbonell et al. 1996; 1999a; Dennell 1998; Dennell y Roebroeks 1996; Roebroeks y van Kolfschoten 1998; Rolland 1992; Rolland 1998). Sin entrar a fondo en estas cuestiones, que darían lugar a otro artículo, merece la pena comentar, en líneas generales, algunas de las hipótesis planteadas.

A la luz de las evidencias de la Península Ibérica, Francia e Italia, parece indudable que el poblamiento de Europa se remonta como mínimo a hace algo más de un millón de años (ca. 1,3 ma). Actualmente es insostenible la posición de un poblamiento no anterior a los 500,000 años (Roebroeks y van Kolfschoten 1998). Lo que no parece tan claro es cuándo salieron estos homínidos de África. El yacimiento más antiguo fuera de 
África es Dmanisi (Georgia), con fósiles humanos adscritos a Homo ergaster, industria lítica de Modo 1 y una rica fauna (Gabunia et al. 2000). La cronología de Dmanisi se situaría alrededor de 1,6 millones de años, en función de las dataciones paleomagnéticas y de la fauna (Gabunia et al. 2000; Goguitchaichvili y Parés 2000). En Próximo Oriente el yacimiento de 'Ubeidiya tiene industria de Modo 2 con una datación de ca. 1,3-1,4 ma. (en función de la fauna) (Bar-Yosef y Belfer-Cohen 2001). Por tanto, podría hablarse de como mínimo dos "oleadas" migratorias anteriores a hace 1 millón de años. Dado que las industrias más antiguas de Europa (incluidas las de la Península Ibérica) son de Modo 1, parecería más lógico relacionar a los pobladores de Europa con los homínidos de Dmanisi que con los de 'Ubeidiya. Sin embargo, existe un lapso cronológico muy notable entre la cronología generalmente aceptada para Dmanisi y la de los yacimientos más antiguos de Europa. La explicación alternativa consistiría en un poblamiento de Europa directamente desde África, y no a través del Próximo Oriente y Europa oriental. La evidencia arqueológica más antigua del norte de África se encuentra en Ain Hanech, con industria de Modo 1 datada en 1,8 millones de años (Sahnouni 1998; Sahnouni y Heinzelin 1998). En Marruecos la crítica a los conjuntos antiguos con cantos tallados, adscritos al Pleistoceno inferior, ha llevado a algunos investigadores a proponer que la industria más antigua es de Modo 2 (yacimientos de las canteras Thomas y Oulad-Hamida), y no va más allá del Pleistoceno inferior final (Raynal et al. 2001). Sin embargo, teniendo en cuenta la cercana presencia de Ain Hanech, resulta difícil descartar de entrada cualquier evidencia de Modo 1 en Marruecos, anterior a las industrias achelenses. De esta manera, cabe plantear la posibilidad de un acceso a la Península a través del estrecho de Gibraltar.

Con el nivel del mar actual la distancia más corta entre los dos litorales separados por el estrecho de Gibraltar es de 14 kilómetros. Si tomamos como referencia las isobatas de $100 \mathrm{~m}$ esta distancia se reduce a $10 \mathrm{~km}$ entre las zonas de Tarifa y Tánger. Según Martinet y Searight (Martinet y Searight 1994) si el nivel del mar descendiera 100 metros aparecerían algunas pequeñas islas en el estrecho, en el sector entre Punta Paloma y Tánger. De esta manera habría diversos tramos de navegación, ninguno de los cuales superaría los $5 \mathrm{~km}$. Las distancias podrían haber sido incluso más cortas bajo condiciones totalmente glaciales, con una regresión de 120 o 130 metros. Claro que deberíamos asumir una estabilidad tectónica y de los sedimentos depositados en el fondo del estrecho a lo largo del Pleistoceno (Straus 2001). Tampoco sabemos hasta qué punto las corrientes marinas eran diferentes a las actuales bajo unas condiciones glaciales.

No se puede descartar por completo el paso a través del estrecho de Gibraltar durante el Pleistoceno inferior (Giles y Santiago 1987), pero hubiera debido ser algo más que un acontecimiento puntual, sólo de esta manera se explicaría la existencia de los yacimientos que hemos comentado anteriormente. Puede aceptarse que en circunstancias excepcionales algunos humanos cruzaran el estrecho, pero no es fácil suponer que lo hicieran repetidamente. Es precisamente esta circunstancia lo que hace difícil explicar un poblamiento inicial realizado exclusiva o fundamentalmente a través de Gibraltar. El paso de contingentes de población suficientemente significativos sería menos problemático a través del Próximo Oriente, aunque la distancia sea más larga. Por otra parte, es más factible un paso más intenso de población por Gibraltar en época más reciente, a inicios del Pleistoceno medio, hace alrededor de 600-500 ka. De esta manera se podrían explicar las similitudes entre el Modo 2 de la Península y del norte de África, tantas veces comentado por diversos investigadores (Aguirre y Carbonell 2001; Alimen 1975; Giles y Santiago 1987).

\section{AGRADECIMIENTOS}

El proyecto de investigación de Atapuerca es financiado por el Ministerio de Educación y Ciencia ("Autoecología humana y tecnología de los pobladores prehistóricos de la Sierra de Atapuerca", BXX20001258-C03-03).Las excavaciones cuentan con el apoyo financiero de la Junta de Castilla y León. 


\section{BIBLIOGRAFÍA}

AGUIRRE, E. y CARBONELL, E. (2001): "Early human expansions into Eurasia: The Atapuerca evidence", Quaternary International 75: 11-18.

ALIMEN, H. (1975): "Les «isthmes» hispano-marocain et siculo-tunisien aux temps acheuleens", L'Anthropologie 79(3): 399-436.

ARRIBAS, A. y PALMQVIST, P. (1999a): "On the Ecological Connection Between Sabre-tooths and Hominids: Faunal Dispersal Events in the Lower Pleistocene and a Review of the Evidence for the First Human Arrival in Europe", Journal of Archaeological Science 26: 571-585.

ARRIBAS, A. y PALMQVIST, P. (1999b): "Yacimientos paleontológicos de Orce-Venta Micena", en J. J. Durán y R. Nuche (eds), El Patrimonio Geológico de Andalucía: 206-211. Madrid, ENRESA.

BAR-YOSEF, O. y BELFER-COHEN, A. (2001): "From Africa to Eurasia -early dispersals", Quaternary International 75: 19-28.

BERMÚDEZ DE CASTRO, J. M.; ARSUAGA, J. L.; CARBONELL, E.; ROSAS, A.; MARTÍNEZ, I. y MOSQUERA, M. (1997): "A hominid from the lower Pleistocene of Atapuerca, Spain: Possible ancestor to Neanderthals and modern humans", Science 276(5317): 1392-1395.

BONIFAY, E. (1991): "Les premières industries du Sud-est de la France et du Massif-Central", en E. Bonifay y B. Vandermeersch (eds), Les Premiers Européens: 63-80. Paris, Editons du C.T.H.S.

BONIFAY, E.; BONIFAY, M. F.; PANATTONI, R. y TIERCELIN, J. J. (1976): "Soleihac (Blanzac, Haute Loire), nouveau site préhistorique au début du Pléistocène moyen", B.S.P.F. 73: 293-304.

BOTELLA, M. C.; VERA, J. A. y PORTA, J. D. (1975): "El yacimiento Achelense de Solana del Zamborino, Fonelas (Granada)", Cuadernos de Prehistoria de la Universidad de Granada 1: 1-46.

BRACCO, J.-P. (1991): "Typologie, technologie et matières premières des industries du très ancien paléolithique en Velay (Massif-Central, France)", en E. Bonifay y B. Vandermeersch (eds), Les Premiers Européens: 93-100. Paris, C.T.H.S.

BREUIL, H. y ZBYSZEWSKI, G. (1942-1945): Contribution à l'étude des industries paléolithiques de Portugal et de leurs rapports avec la géologie du Quaternaire. Tome I: Les principaux gisements des deux rives de l'ancien estuaire du Tage. Communicaçoes dos Serviços Geologicos de Portugal. Vol. 23 \& 26. Lisboa, Serviços Geologicos de Portugal.

CARBONELL, E. (1999): "Cuándo y cómo llegan los homínidos a Europa", en L. Pallí Buxó y C. Roqué Pau (eds), Avances en el estudio del Cuaternario español: 11-18. Girona.

CARBONELL, E. y RODRÍGUEZ, X. P. (1994): "Early Middle Pleistocene deposits and artefacts in the Gran Dolina site (TD4) of the 'Sierra de Atapuerca' (Burgos, Spain)", Journal of Human Evolution 26: 291-311.

CARBONELL, E.; MOSQUERA, M.; OLLÉ, A.; RODRÍGUEZ, X. P.; SALA, R.; VAQUERO, M. y VERGÈS, J. M. (1992): New elements of the logical analytic system. First International Meeting on Technical Systems to Configure Lithic Objects of scarce elaboration (Montblanc, 1992). Cahier Noir. Tarragona, Laboratori d'Arqueologia de la Universitat Rovira i Virgili/ Reial Societat Arqueològica Tarraconense.

CARBONELL, E.; BERMÚDEZDECASTRO, J. M.; ARSUAGA, J.L.; DÍEZ, J.C.; ROSAS, A.; CUENCABESCÓS, G.; SALA, R.; MOSQUERA, M. y RODRÍGUEZ, X.P. (1995): "Lower Pleistocene Hominids and Artifacts from Atapuerca-TD6 (Spain)", Science 269(5225): 826-830.

CARBONELL, E.; MOSQUERA, M.; RODRÍGUEZ, X. P. y SALA, R. (1996): "The First Human Settlement of Europe", Journal of Anthropological Research 52: 107-114.

CARBONELL, E.; OLLÉ, A.; RODRÍGUEZ, X. P.; SALA, R. y VERGÈS, J. M. (1997): "Instrumentos de los homínidos de Atapuerca", Mundo Científico 175: 54-59. 
CARBONELL, E.; ARSUAGA, J. L.; BERMÚDEZ DE CASTRO, J. M.; CÁCERES, I.; DÍEZ, J. C.; FERNÁNDEZ JALVO, Y.; MOSQUERA, M.; RODRÍGUEZ, X. P.; ROSELL, J.; SALA, R. y VALLVERDÚ, J. (1998): "Homo antecessor y su medio natural", Mundo Científico 192: 42-49.

CARBONELL, E.; MOSQUERA, M.; RODRÍGUEZ, X.P.; SALA, R. y VAN DER MADE, J. (1999a): "Out of Africa: The Dispersal of the Earliest Technical Systems Reconsidered", Journal of Anthropological Archaeology 18: 119-136.

CARBONELL, E.; GARCÍA-ANTÓN, M.D.; MALLOL, C.; MOSQUERA, M.; OLLÉ, A.; RODRÍGUEZ, X.P.; SAHNOUNI, M.; SALA, R. y VERGÈS, J. M. (1999b): "The TD6 level lithic industry from Gran Dolina, Atapuerca (Burgos, Spain): production and use", Journal of Human Evolution 37: 653-693.

CARBONELL, E.; ESTEBAN, M.; NÁJERA, A. M.; MOSQUERA, M.; RODRÍGUEZ, X. P.; OLLÉ, A.; SALA, R.; VERGĖS, J. M.; BERMÚDEZ DE CASTRO, J. M. y ORTEGA, A. I. (1999c): "The Pleistocene site of Gran Dolina, Sierra de Atapuerca, Spain: a history of the archaeological investigations", Journal of Human Evolution 37: 313-324.

CARBONELL, E.; RODRÍGUEZ, X. P.; SALA, R. y LOZANO, M. (2000): "Atapuerca y los modos técnicos de producción lítica del Pleistoceno inferior y medio", en L. Caro Dobón, H. Rodríguez Otero, E. Sánchez Compadre, B. López Martínez y M. J. Blanco (eds), Tendencias actuales de la Investigación en la Antropología Física Española: 19-29. León, Secretariado de Publicaciones, Universidad de León.

CUENCA-BESCÓS, G.; CANUDO, J. I. y LAPLANA, C. (1999): "Lower Pleistocene arvicolids (Rodentia, Mammalia) of Trinchera Dolina 6 (Atapuerca, Burgos, Spain)", en J. I. Canudo y G. Cuenca-Bescós (eds), IV European Workshop on Vertebrate Paleontology (Albarracín, Teruel, Spain). Abstracts: 42-43.

DENNELL, R. (1998): "The TD6 horizon of Atapuerca and the Earliest Colonisation of Europe: a Eurasian perspective", en E. Carbonell, J. M. Bermúdez De Castro, J. L. Arsuaga y X. P. Rodríguez (eds), Los primeros pobladores de Europa: Últimos descubrimientos y debate actual: 75-97. Burgos, Diario de Burgos / Caja de Burgos.

DENNELL, R. y ROEBROEKS, W. (1996): "The earliest colonization of Europe: the short chronology revisited", Antiquity 70(269): 535-542.

DÍEZ, J. C.; FERNÁNDEZ-JALVO, Y.; ROSELL, J. y CÁCERES, I. (1999): "Zooarchaeology and taphonomy of Aurora Stratum (Gran Dolina, Sierra de Atapuerca, Spain)", Journal of Human Evolution 37(3-4): 623-652.

FALGUÈRES, C.; BAHAIN, J.-J.; YOKOYAMA, Y.; ARSUAGA, J. L.; BERMÚDEZ DE CASTRO, J. M.; CARBONELL, E.; BISCHOFF, J. L. y DOLO, J.-M. (1999): "Earliest humans in Europe: the age of TD6 Gran Dolina, Atapuerca, Spain", Journal of Human Evolution 37(3-4): 343-352.

FERNÁNDEZ-JALVO, Y.; DIEZ, J.C.; BERMÚDEZDECASTRO, J. M.; CARBONELL, E. y ARSUAGA, J. L. (1996): "Evidence of early cannibalism", Science 271(5247): 277-278.

FERNÁNDEZ-JALVO, Y.; DIEZ, J. C.; CÁCERES, I. y ROSELL, J. (1999): "Human cannibalism in the Early Pleistocene of Europe (Gran Dolina, Sierra de Atapuerca, Burgos, Spain)", Journal of Human Evolution 37(3-4): 591-622.

GABUNIA, L.; VEKUA, A.; LORDKIPANIDZE, D.; SWISHER III, C. C.; FERRING, R.; JUSTUS, A.; NIORADZE, M.; TVALCHRELIDZE, M.; ANTÓN, S. C.; BOSINSKI, G.; JÖRIS, O.; LUMLEY, M.-A. D.; MAJSURADZE, G. y MOUSKHELISHCILI, A. (2000): "Earliest Pleistocene Hominid Cranial Remains from Dmanisi, Republic of Georgia: Taxonomy, Geological Setting, and Age", Science 288: 1019-1025.

GAMBLE, C. (1994): "Time for Boxgrove man", Nature 369(6478): 275-276.

GARCÍA ANTÓN, M. (1998): "Reconstrucciones de paleovegetación en Atapuerca según análisis polínico", en E. Aguirre (ed.), Atapuerca y la Evolución humana: 61-71. Madrid, Fundación Ramón Areces.

GARCÍA, N. y ARSUAGA, J. L. (1999): "Carnivores from the Early Pleistocene hominid-bearing Trinchera Dolina 6 (Sierra de Atapuerca, Spain)", Journal of Human Evolution 37(3-4): 415-430. 
GIBERT, J.; GIBERT, L.; IGLESIAS, A. y MAESTRO, E. (1998a): "Two "Oldowan" assemblages in the Plio-Pleistocene deposits of the Orce region, southeast Spain", Antiquity 72: 17-25.

GIBERT, J.; CAMPILLO, D.; ARQUÉS, J. M.; GARCÍA-OLIVARES, E.; BORJA, C. y LOWENSTEIN, J. (1998b): "Hominid status of the Orce cranial fragment reasserted", Journal of Human Evolution 34: 203-217.

GILES, F. y SANTIAGO, A. (1987): "El poblamiento del sur de la Península Ibérica en el Pleistoceno inferior a través de Gibraltar", en Actas del Congreso Internacional «El estrecho de Gibraltar», Ceuta 1987. Tomo I, Prehistoria e Historia de la Antigüedad: 97-109. Ceuta.

GILES, F.; CANO, J. S.; SANTIAGO, A.; GUTIÉRREZ, J. M.; MATA, E.; GRACIA, J.; AGUILERA, L. y PRIETO, O. (2000): "Poblamiento paleolítico en la Cuenca media-baja del río Miño. Sector La Guardia-Tuy (Pontevedra)- Cortegada (Orense). Secuencia cronoestratigráfica", en R. Balbín, N. Bicho, E. Carbonell, B. Hackett, A. Moure, L. Raposo, M. Santonja y G. Vega (eds), Paleolitico da Península Ibérica. Actas do $3^{\circ}$ Congresso de Arqueología Penínsular (Vila Real, 1999): 101-121. Porto, ADECAP.

GOGUITCHAICHVILI, A. y PARÉS, J. M. (2000): "A recognition palaeomagnetic study of volcanic and sedimentary rocks from Dmanissi (Caucasus): implication for the oldest human occupation in Europe", C.R. Acad. Sci. Paris, Sciences de la Terre et des planètes 331: 183-186.

LUMLEY, H. D.; FOURNIER, A.; KRZEPKOWSKA, J. y ECHASOUX, A. (1988): "L'industrie du Pleistocene inférieur de la grotte du Vallonet, Roquebrune-Cap-Martin, Alpes Maritimes", L'Anthropologie 92: 501-614.

MADE, J. V. D. (1998): "Ungulates from Gran Dolina (Atapuerca, Burgos, Spain)", Quaternaire 9(4): 267-281.

MADE, J. V.D. (1999): "Ungulates from Atapuerca TD6", Journal of Human Evolution 37(3-4): 389-413.

MARTINET, G. y SEARIGHT, S. (1994): "Le Maghreb préhistorique et la navigation", Bulletin de la Société d'Études et de Recherches Préhistoriques des Eyzies 43: 85-111.

MARTÍNEZ NAVARRO, B.; TURQ, A.; AGUSTÍ BALLESTER, J. y OMS, O. (1997): "Fuente Nueva 3 (Orce, Granada, Spain) and the first human occupation of Europe", Journal of Human Evolution 33: 611-620.

MOYÀ-SOLÀ, S. y KÖHLER, M. (1997): "The Orce skull: anatomy of a mistake", Journal of Human Evolution 33(1): 91-97.

OMS, O.; AGUSTÍ, J.; GABÀS, M. y ANADÓN, P. (2000a): "Lithostratigraphical correlation of micromammal sites and biostratigraphy of the Upper Pliocene to Lower Pleistocene in the Northeast Guadix-Baza Basin (southern Spain)", Journal of Quaternary Science 15(1): 43-50.

OMS, O.; PARÉS, J. M.; MARTÍNEZ-NAVARRO, B.; AGUSTÍ, J.; TORO, I.; MARTÍNEZ-FERNÁNDEZ, G. y TURQ, A. (2000b): "Early human occupation of Western Europe: Paleomagnetic dates for two paleolithic sites in Spain", Proc Natl Acad Sci U S A 97(19): 10666-10670.

PALMQVIST, P. (1997): "A critical re-evaluation of the evidence for the presence of hominids in Lower Pleistocene times at Venta Micena, Southern Spain", Journal of Human Evolution 33(1): 83-89.

PALMQVIST, P.; PÉREZ-CLAROS, J. A.; GIBERT, J. y SANTAMARÍA, J. L. (1996): "Comparative Morphometric Study of a Human Phalanx from the Lower Pleistocene Site at Cueva Victoria (Murcia, Spain), by means of Fourier Analysis, Shape Coordinates of Landmarks, Principal and Relative Warps", Journal of Archaelogical Science 23: 95-107.

PARÉS, J. M. y PÉREZ-GONZÁLEZ, A. (1995): "Paleomagnetic age for hominid fossils at Atapuerca archaeological site, Spain", Science 269(5225): 830-832.

PARÉS, J. M. y PÉREZ-GONZÁLEZ, A. (1999): "Magnetochronology and stratigraphy at Gran Dolina section, Atapuerca (Burgos, Spain)", Journal of Human Evolution 37(3-4): 325-342. 
PERETTO, C., ed. (1994): Le industrie lithiche del giacimento paleolitico di Isernia La Pineta, la tipologia, le tracce di utilizzazione, la sperimentazione.. Cosmo Iannone Editore: Isernia.

PERETTO, C., ed. (1996): I Reperti paleontologici del Giacimento Paleolitico di Isernia La Pineta: L'Uomo e l'Ambiente. Istituto Regionale per Gli Studi Storici del Molice "V. Cuoco": Isernia.

PERETTO, C.; AMORE, F. O.; ANTONIAZZI, A.; ANTONIAZZI, A.; BAHAIN, J. J.; CATTANI, L.; CAVALLINI, E.; ESPOSITO, P.; FALGUERES, C.; GAGNEPAIN, J.; HEDLEY, I.; LAURENT, M.; LEBRETON, V.; LONGO, L.; MILLIKEN, S.; MONEGATTI, P.; OLLE, A.; PUGLIESE, A.; RENAULTMISKOVSKY, J.; SOZZI, M.; UNGARO, S.; VANNUCI, S.; VERGÈS, J. M.; WAGNER, J. J. y YOKOYAMA, Y. (1998): "Industrie lithique de Ca'Belvedere di Monte Poggiolo: Stratigraphie, matière première, typologie, remontages et traces d'utilisation", L'Anthropologie 102: 343-465.

RAPOSO, L. y CARREIRA, J. R. (1986): "Acerca da existência de complexos industriais pré-acheulenses no território português", O Arqueólogo Português 4(4): 7-90.

RAPOSO, L. y SANTONJA, M. (1995): "The earliest occupation of Europe: the Iberian peninsula", en W. Roebroeks y T. Van Kolfschoten (eds), The earliest occupation of Europe: 7-25. Leiden, University of Leiden.

RAYNAL, J.-P.; SBIHI-ALAOUI, F. Z.; GERAADS, D.; MAGOGA, L. y MOHI, A. (2001): "The earliest occupation of North-Africa: the Moroccan perspective", Quaternary International 75: 65-75.

RODRÍGUEZ ÁLVAREZ, X. P. (1997): Los Sistemas Técnicos de Producción Lítica del Pleistoceno Inferior y Medio en la Península Ibérica: Variabilidad Tecnológica entre Yacimientos del Noreste y de la Sierra de Atapuerca. Tesis Doctoral, Departament d'Història i Geografia. Tarragona: Universitat Rovira i Virgili.

RODRÍGUEZ, J. (1997): Análisis de la estructura de las comunidades de mamíferos de Pleistoceno de la Sierra de Atapuerca. Revisión de metodologías.. Tesis Doctoral. Madrid: Universidad Autónoma de Madrid. 353 pp.

ROEBROEKS, W. y VAN KOLFSCHOTEN, T. (1994): "The Earliest Occupation of Europe: A Short Chronology", Antiquity 68: 489-503.

ROEBROEKS, W. y VAN KOLFSCHOTEN, T. (1998): "The earliest occupation of Europe: a view from the North", en E. Aguirre (ed.), Atapuerca y la evolución humana: 153-168. Madrid, Fundación Ramón Areces.

ROEBROEKS, W.; CONARD, N. J. y VAN KOLFSCHOTEN, T. (1992): "Dense Forest, Cold Steppes, and the Palaeolithic Settlement of Northern Europe", Current Anthropology 33(5): 551-586.

ROJO, A. y MORENO, M. A. (1979): "Industrias del Paleolítico inferior en las terrazas del Pisuerga (Valladolid)", Boletín de Estudios de Arte y Arqueología de la Universidad de Valladolid 45: 148-157.

ROLLAND, N. (1992): "The palaeolithic colonization of Europe: an archaeological and biogeographic perspective", Trabajos de Prehistoria 49: 69-111.

ROLLAND, N. (1998): "The Lower Palaeolithic settlement of Eurasia, with special reference to Europe", en M. D. Petraglia y R. Korisettar (eds), Early Human Behaviour in Global Context: 187-220. London, Routledge.

ROSELL, J. (1998): "Les premières occupations humaines à la Sierra de Atapuerca (Burgos, Espagne) les niveaux TDW4 et TDW-4B", en, Économie Préhistorique: les comportements de subsitance au Paléolithique. XVIIIe Rencontres Internationales d'Archéologie et d'Histoire d'Antibes: 154-161. Sophia Antipolis, Éditions APDCA.

ROSELL, J.; CÁCERES, I. y HUGUET, R. (1998): "Systèmes d'occupation anthropique pendant le Pléistocene inférieur et moyen à la Sierra de Atapuerca (Burgos, Espagne)", Quaternaire 9(4): 355-360.

RUIZ BUSTOS, A. (1984): "El yacimiento paleontológico de Cúllar Baza I", Investigación y Ciencia 91: 20-26. 
SAHNOUNI, M. (1998): The Lower Palaeolithic of the Maghreb: Excavations and Analyses at Ain Hanech, Algeria. Cambridge Monographies in African Archaeology 42. Oxford, BAR International Series 689.

SAHNOUNI, M. y HEINZELIN, J. D. (1998): "The site of Ain Hanech revisited: New investigations at this Lower Pleistocene site in northern Algeria", Journal of Archaeological Science 25(11): 1083-1101.

SALA, R. (1997): Formes d'ús i criteris d'efectivitat en conjunts de Mode 1 i Mode 2: Anàlisi de les deformacions per ús dels instruments lítics del Plistocè inferior (TD6) i mitjà (TG11) de la Sierra de Atapuerca. Ph. D. Thesis, Dept. Història i Geografia. Tarragona: Universitat Rovira i Virgili.

SALA, R. (1998): "The Use and Efficiency of a Mode 1 Lithic Production System", en A. Antoniazzi, A. Antoniazzi, J. L. Arsuaga, J. M. Bermúdez De Castro, E. Carbonell, E. Cavallini, S. D. Lernia, F. Fontana, C. Gutiérrez, L. Longo, G. Manzi, S. Milliken, L. Oosterbeek, V. Pavukova, M. Persani, V. Pesce, K. Pizchelauri, L. Prati, R. Sala y C. Peretto (eds), Proceedings of the XIII International Congress of Prehistoric and Protohistoric Sciences. September 8-14, 1996. Volume 6, Tome II.: 1303-1310. Forli, ABACO.

SÁNCHEZ-MARCO, A. (1999): "Implications of the avian fauna for paleoecology in the Early Pleistocene of the Iberian Peninsula", Journal of Human Evolution 37(3-4): 375-388.

SANTONJA, M. y PÉREZ-GONZÁLEZ, A. (1997): "Los yacimientos achelenses en terrazas fluviales de la Meseta Central española", en J. Rodríguez Vidal (ed.), Cuaternario Ibérico: 224-234. Huelva, Asociación Española para el Estudio del Cuaternario.

STRAUS, L. G. (2001): "Africa and Iberia in the Pleistocene", Quaternary International 75: 91-102.

TORO, I. (1999): "El proyecto de investigación sobre el Plio-pleistoceno en la cuenca de Guadix-Baza: De la investigación al desarrollo. Hacia un modelo de gestión del patrimonio arqueológico y paleontológico", Boletín del Instituto Andaluz del Patrimonio Histórico 29: 156-167.

TURQ, A.; MARTÍNEZ-NAVARRO, B.; PALMQVIST, P.; ARRIBAS, A.; AGUSTÍ, J. y RODRÍGUEZ VIDAL, J. (1996): "Le Plio-Pleistocene de la région d'Orce, province de Grenade, Espagne: bilan et perspectives de recherche", Paleo 8: 161-204.

VALLESPÍ PÉREZ, E. (1994): "El Bajo Guadalquivir en el Paleolítico Inferior y Medio peninsular", en, Homenaje a González Echegaray: 13-16. Santander, Museo y Centro de Investigación de Altamira. 